\title{
Nivel de dolor y elección de analgesia en el parto determinada por la realización de educación maternal
}

\author{
Juan Miguel Martínez G. 1,2,3 Miguel Delgado R. 2,3 \\ 1 Servicio Andaluz de Salud, ${ }^{2}$ Universidad de Jaén, ${ }^{3}$ Centro de Investigación Biomédica en Red de Epidemiología y \\ Salud Pública (CIBERESP). Andalucía, España.
}

Esta investigación ha sido subvencionada por el Fondo de Investigación Sanitaria del Instituto de Salud Carlos III (PI11/01388)

\section{RESUMEN}

Objetivo: Conocer la influencia de la educación maternal sobre el nivel de dolor que manifiesta la mujer durante el proceso de parto, la utilización de analgesia epidural y el uso de medidas analgésicas alternativas. Método: Estudio multicéntrico observacional, realizado en cuatro hospitales de Andalucía desde enero de 2011 a enero de 2012. La población estudio fueron mujeres primíparas que tuvieron su parto en alguno de estos centros. Se estudiaron diferentes variables sociodemográficas, nivel de dolor y uso de medidas analgésicas. Los datos se recogieron a través de una entrevista y de la historia clínica. En el análisis se estimaron Odds Ratios (OR) y comparación de medias, crudas y ajustadas. Resultados: Participaron en el estudio 520 mujeres. No se detectó asociación entre la realización de educación maternal por parte de la mujer y el nivel de dolor que esta padeció durante el proceso de parto $(p>0,05)$. Tampoco se detectó influencia con la utilización de medidas analgésicas alternativas $(\mathrm{ORa}=1,14$; IC95\%: 0,70-1,83), ni de la analgesia epidural (ORa= 1,58; IC95\%: 0,96-2,63). Conclusión: La educación maternal no es eficaz para el control del dolor que produce el proceso de parto, ni determina el uso de otras medidas analgésicas.

\section{PALABRAS CLAVE: Educación maternal, parto, dolor, analgesia epidural, medidas analgésicas alternativas}

\section{SUMMARY}

Objective: To determine the influence of maternal education on the level of pain expressed by women during the delivery process, the use of epidural analgesia and analgesic use of alternative measures. Methods: A multicenter observational conducted in four hospitals in Andalusia from January 2011 to January 2012. The study population consisted of primiparous women who gave birth in one of these centers. Sociodemographic variables were studied, level of pain and use of analgesic measures. The data were collected through an interview and medical history. The analysis estimated the Odds Ratios (OR) and mean, crude and adjusted. Results: The study involved 520 women. No association was found between maternal education being carried out by women and that this level of pain endured during the delivery process $(p>0.05)$. Nor influence was detected with the use of alternative analgesic measures $(\mathrm{aOR}=1.14 ; 95 \% \mathrm{Cl}$ : $0.70-1.83)$, or epidural analgesia (aOR=1.58; 95\% Cl: $0.96-2.63)$. Conclusion: The maternal education is not effective for pain control that produces the delivery process, or determine the use of other analgesic measures.

KEY WORDS: Maternal education, labor, pain, epidural analgesia, analgesic action alternatives 


\section{INTRODUCCIÓN}

El dolor asociado al parto ha sido descrito como una de las formas más intensas de dolor que puede experimentarse. El dolor experimentado por las mujeres durante el parto es producido por las contracciones uterinas, la dilatación del cuello del útero y al final de la primera etapa y durante la segunda, por la dilatación de la vagina y del piso pelviano para acomodar al feto (1).

El dolor del parto es agudo y de una gran variabilidad. El $20 \%$ de las parturientas describen la intensidad del dolor como insoportable, el 30\% como severo, el $35 \%$ como moderado y tan sólo el $15 \%$ señalan la intensidad como mínima (2).

El control del dolor es uno de los aspectos a llevar a cabo por los profesionales implicados en el proceso de parto y de lo que más preocupa a las mujeres. Existen múltiples métodos para el control de ese dolor aunque no todos han demostrado su eficacia. Entre las medidas para el control de ese dolor se encuentra la analgesia epidural, la educación maternal, el apoyo durante el parto, las inyecciones dérmicas de suero estéril y la inmersión en agua (3).

El embarazo y el parto es uno de los acontecimientos más importantes en la vida de una mujer, tanto por las circunstancias emocionales asociadas como por las consecuencias que para ella y el recién nacido se podrían derivar. El modelo de atención al parto ha experimentado un cambio importante en los últimos años pasando de un modelo medicalizado a un modelo menos intervencionista (4).

Durante el parto, además o en lugar de la analgesia, la mujer puede controlar el dolor mediante una serie de estrategias de afrontamiento. La educación maternal brinda la oportunidad a las mujeres a prepararse para un parto menos doloroso $(5,6)$. Sin embargo los estudios sobre el efecto de la educación maternal en el dolor de la mujer durante el parto son escasos y arrojan resultados incongruentes (5-8).

El programa de educación maternal forma parte de la cartera de servicios del sistema sanitario público andaluz, siendo de acceso universal y gratuito. El programa consta de 10 sesiones estandarizadas que se desarrolla de forma grupal a partir de las 28 semanas de gestación. Estas sesiones consta tanto de charlas teóricas como ejercicio físico que versan, entre otros temas, en la preparación física y psicológica para el parto con actividades como visualización de videos de parto, ejercicios de pujo, relajación, respiración, etc. Se establece como criterio de calidad la asistencia de al menos 3 sesiones, es decir, con este número de sesiones la administración sanitaria considera que una mujer ha realizado educación maternal (9).

Ante este nuevo contexto en la atención al parto, los escasos estudios y los resultados incongruentes de estos, se propuso el objetivo de conocer la influencia de la educación maternal sobre el nivel de dolor que manifiesta la mujer durante el proceso de parto, la utilización de analgesia epidural y el uso de medidas analgésicas alternativas.

\section{PACIENTES Y MÉTODO}

Se realizó un estudio observacional entre enero de 2011 y enero de 2012 en las áreas sanitarias de la provincia de Jaén (Complejo Hospitalario Universitario de Jaén y Hospital San Juan de la Cruz de Úbeda), Hospital de Poniente en El Ejido (Almería) y en el Hospital Universitario Virgen de las Nieves de Granada. La población de referencia fueron mujeres que dieron a luz en algunos de estos centros que fueran primíparas, de gestación única, y mayor de 18 años. El estudio fue aprobado por los Comités de Ética de los centros respectivos y se pidió consentimiento informado. Se fijó como criterio de exclusión la barrera idiomática.

Se recogió información sobre datos sociodemográficos (edad, sexo, estado civil, nacionalidad, nivel de ingresos, nivel máximo de estudios alcanzados, realización de trabajo durante el embarazo, tipo de contrato, sector en el que trabaja, raza y nacionalidad), variables relacionadas con la educación maternal. Para medir el nivel de dolor se utilizó la escala numérica de intensidad de dolor [0 a 10]. Otorgando puntuación 0 a sin dolor, 5 a un dolor moderado y 10 el peor dolor posible (10). También se recogieron variables relacionadas con la utilización de la analgesia epidural y de medidas analgésicas alternativas.

Los datos se recogieron a través de una entrevista y fueron validados con la historia clínica y cartilla maternal. El cuestionario constaba de 140 ítems (130 cerradas y 10 abiertas). Fue aplicado por 24 entrevistadores adiestrados. Las mujeres se seleccionaron de forma consecutiva.

En el análisis de datos, para variables dicotómicas se evaluó según Odds Ratio (OR) con un intervalo de confianza del 95\% (IC95\%). En el análisis multivariable se aplicó la regresión logística, reteniendo como confundidores a las variables que alteraron el coeficiente de la exposición principal en más del $10 \%$. Como posibles confundidores a priori se consideró las características sociodemográficas de la mujer y la presencia de patología durante el embarazo. Cuando la variable resultado fue continua (ej., nivel de dolor en el parto), se utilizó la comparación de medias, y en el análisis multivariable se utilizó el análisis de la covarianza, ajustando por las mismas variables mencionadas.

\section{RESULTADOS}

Participaron en el estudio 520 mujeres, tenían una media de edad de 29,91 $\pm 5,30$ años. Raza blanca $97,88 \%$, casadas $64,7 \%$, y $89,62 \%$ eran de nacionalidad española. El $31,73 \%$ de estas mujeres 
poseían estudios universitarios y el $46,94 \%$ disponía de ingresos mensuales entre 1.000-1.999 €. La mayoría de estas mujeres estaban sanas antes del embarazo y en el $90 \%$ de los casos el embarazo fue buscado. El $77,50 \%$ de las mujeres controlaron su embarazo en atención primaria, el $91,80 \%$ tenían un buen control prenatal y el $68,65 \%$ acudió al programa de educación maternal.

Las mujeres que realizaron educación maternal otorgaron una media de 7,08(IC95\%: 6,84-7,32) sobre 10 al nivel de dolor que padeció en el parto frente al 7,31(IC95\%: 6,92-7,71) $(p=0,297)$ al ajustar por estado civil materno, nivel de estudios máximos alcanzados, presencia de patología en el embarazo, embarazo buscado, edad materna, utilización de medicación en el periodo de dilatación, utilización de analgesia epidural y de medidas analgésicas no farmacológicas durante el parto, no se produjo cambio significativo $(p=0,690)$.

En la Tabla I se puede comprobar como la educación maternal no tuvo influencia en la utilización de la analgesia epidural (ORa=1,58; IC95\%: 0,96$2,63)$, ni el uso de medidas analgésicas alternativas (ORa=1,14; IC95\%: 0,70-1,83). Si bien hay que tener en cuenta que el $15,15 \%$ ( $n: 25)$ de las mujeres que no asistió a educación maternal no se les administró la analgesia epidural porque ese era su deseo y no la solicitaron, sin embargo este porcentaje fue del 7,63\% (n: 27) en el grupo de mujeres que sí acudieron al programa de educación maternal. La contraindicación médica para el uso de la epidural fue el motivo de no utilizarla para el 3,03\% (n: 5) de las mujeres que no acudieron frente al 2,26\% (n: 8) de las que asistieron a la educación maternal. Hubo otras razones como la saturación del servicio, expulsivo inminente, etc., para la no administración de la analgesia epidural en el 5,45\% (n: 9) de las mujeres que no había realizado la educación maternal versus $5,93 \%$ ( $n: 21)$ en las mujeres que si habían asistido a las sesiones de educación maternal $(p=0,057)$.

Las mujeres que utilizaron la analgesia epidural terminaron en un 49,29\% (n: 209) en parto eutócico, en un $32,31 \%$ ( $n: 137$ ) en parto instrumental y en un $18,40 \%$ ( $n: 78$ ) en cesárea; mientras que las mujeres que no usaron la analgesia epidural finalizaron en un $64,21 \%$ ( $n: 61)$ en eutócico, en un 18,95\% ( $n$ : 18) en parto instrumental y en un $16,84 \%$ (n: 16$)$ en cesárea $(p=0,018)$.

\section{DISCUSIÓN}

Diversos estudios han hallado una asociación positiva entre la realización de educación maternal y el menor nivel de dolor expresado por la madre durante el parto $(5,6)$. Otras investigaciones $(7,8)$ no establecieron ninguna asociación entre ambos parámetros, al igual que en este estudio. Si bien la puntuación media que otorgaron las mujeres que realizan educación maternal al nivel de dolor es sensiblemente menor que las mujeres que no realizaron educación maternal, no se alcanzó significación estadística al $5 \%$. Por lo tanto, en este sentido se está en la línea de las recomendaciones de la Sociedad Española de Ginecología y Obstetricia (3) que afirma que la educación maternal es un método analgésico de eficacia poco demostrada.

Tabla I

ASOCIACIÓN ENTRE LA EDUCACIÓN MATERNAL Y EL USO DE MEDIDAS ANALGÉSICAS DURANTE EL PROCESO DE PARTO

\begin{tabular}{|c|c|c|c|c|c|}
\hline \multirow{2}{*}{ Variable } & \multirow[t]{2}{*}{$\begin{array}{c}\text { Total } \\
\mathrm{n}\end{array}$} & \multicolumn{2}{|c|}{$\begin{array}{c}\text { Educación } \\
\text { maternal }\end{array}$} & \multirow{2}{*}{$\begin{array}{c}\text { Análisis } \\
\text { crudo } \\
\text { OR (IC95\%) }\end{array}$} & \multirow{2}{*}{$\begin{array}{c}\text { Análisis } \\
\text { multivarialble } \\
\text { OR (IC95\%) }\end{array}$} \\
\hline & & $\begin{array}{c}\text { No } \\
\text { n (\%) }\end{array}$ & $\begin{array}{c}\text { Sí } \\
\text { n (\%) }\end{array}$ & & \\
\hline
\end{tabular}

Utilización de analgesia epidural en el parto

\begin{tabular}{lcrrrr} 
No & 95 & $39(41,05)$ & $56(58,95)$ & 1 (referencia) & 1 (referencia) \\
Si & 424 & $126(29,72)$ & $298(70,28)$ & $1,64(1,01-2,66)$ & $1,58(0,96-2,63)$ \\
$\begin{array}{l}\text { Utilización de medidas analgésicas } \\
\text { no farmacológicas en el parto }\end{array}$ & & & & & \\
No & 411 & $131(31,87)$ & $280(68,13)$ & 1 (referencia) & 1 (referencia) \\
Si & 109 & $35(32,11)$ & $74(67,89)$ & $0,98(0,61-1,60)$ & $1,14(0,70-1,83)$ \\
\hline
\end{tabular}

\footnotetext{
* ajustada por estado civil, nivel de estudios máximos alcanzados, presencia de patología del embarazo, embarazo buscado, edad de la madre, modo de inicio de parto, y utilización de medicación en el proceso de dilatación.
} 
En cuanto a la utilización de los métodos analgésicos, no se encontró una asociación entre la realización de la educación maternal y la utilización de la analgesia epidural. Estos resultados coinciden con los de Bergström y cols (11) y Artieta-Pinedo y cols (12), sin embargo Fabian y cols (8) detectaron que las mujeres que realizaban educación maternal utilizaban más la analgesia epidural durante el parto.

En nuestro estudio no se identificó la influencia de la educación maternal sobre el uso de los métodos analgésicos alternativos por parte de la parturienta, resultados que van en la línea opuesta de lo que encontraron Alves y Lima (13) y en contra de los de Soto y cols (14).

La no utilización de analgesia epidural por parte de la mujer durante el proceso de parto fue identificada como un factor protector para la finalización del parto de forma eutócica en concordancia con los resultados de Thorp y cols (15) pero en contra de lo que sugirieron Leighton y cols (16).

De existir un sesgo de selección asociado a la no respuesta, éste habrá tenido una influencia mínima sobre la validez de los resultados, pues no existen razones a priori para pensar que las mujeres que respondieron difieran sustancialmente de las que no respondieron.

No se puede descartar completamente el sesgo de confusión, limitación inherente a la mayoría de los estudios observacionales. Con el nivel de conocimientos existente no es posible realizar un ensayo clínico por razones éticas. A priori no hay fundamento para pensar que no se hayan tenido en cuenta todos los factores de confusión que pudiese alterar algunas de las variables estudiadas. Por ejemplo: la utilización de la analgesia epidural durante el parto, hay hechos que demuestran que la utilización de oxitocina exógena es más dolorosa (17). La administración de oxitocina exógena durante el proceso de parto podría llevar a un aumento del dolor percibido por la mujer, y obligara a demandar la analgesia epidural fue tenido en cuenta en el análisis de datos.

\section{CONCLUSIÓN}

Se puede afirmar que en nuestra experiencia la educación maternal no fue eficaz para el control del dolor que produce el proceso de parto ni determina el uso de otras medidas analgésicas.

Agradecimientos: A todas las mujeres que han participado de manera voluntaria y desinteresada en el estudio, así como el personal que ha participado en la recogida de datos en los distintos centros sanitarios.

\section{REFERENCIAS}

1. Smith CA, Collins CT, Cyna AM, Crowther CA. Tratamientos complementarios y alternativos para el manejo del dolor durante el trabajo de parto (Revisión Cochrane traducida). En: La Biblioteca Cochrane Plus, 2008 Número 2. Oxford: Update Software Ltd. Disponible en: http://www.update-software.com. (Traducida de The Cochrane Library, 2008 Issue 2. Chichester, UK: John Wiley \& Sons, Ltd.).

2. Bonica JJ, McDonald JS. The pain and childbirth. En: Bonica JJ. The management of pain. 2nd edition. Philadelphia: Lea \& Febiger; 1990: pp 1313-43.

3. Sociedad Española de Ginecología y Obstetricia (SEGO). Documento de consenso. Analgesia en el parto. 2006.

4. Ministerio de Sanidad y Consumo. Estrategia de atención al parto normal en el Sistema Nacional de Salud. $1^{\mathrm{a}}$ ed. Madrid: Ministerio de Sanidad y Consumo, 2007.

5. Escott D, Slade P, Spiby H. Preparation for pain management during childbirth: the psychological aspects of coping strategy development in antenatal education. Clin Psychol Rev 2009;29:617-22.

6. Ip WY, Tang CS, Goggins WB. An educational intervention to improve women's ability to cope with childbirth. J Clin Nurs 2009;18:2125-35.

7. Molina Salmerón M, Martínez García AM, Martínez García FJ, Gutiérrez Luque E, Sáez Blázquez R, Escribano Alfaro PM. Impacto de la Educación Maternal, vivencia subjetiva materna y evolución del parto. Rev Enfermería 1996;6:20-9.

8. Fabian HM, Radestad IJ, Waldesnström U. Childbirth and parenthood education classes in Sweden 2005. Women's opinion and possible outcomes. Acta Obstet Gynecol Scand 2005;84:436-43.

9. Junta de Andalucía. Consejería de Salud. Proceso Asistencial Integrado Embarazo, Parto y Puerperio. 2ed. Sevilla: Consejería de Salud, 2005.

10. Pardo $P$, Muñoz T, Chamorro C. Monitorización del dolor. Recomendaciones del grupo de trabajo de analgesia y sedación de la SEMICYUC. Med Intensiva 2006;30:379-85.

11. Bergström $M$, Kieler $H$, Waldenström U. Effects of natural childbirth preparation versus standard antenatal education on epidural rates, experience of childbirth and parental stress in mothers and fathers: a randomized controlled multicentre trial. BJOG 2009;116:116776.

12. Artieta-Pinedo I, Paz-Pascual C, Grandes G, RemiroFernandez de Gamboa G, Odriozola-Hermosilla I, Bacigalupe A, Payo J. The benefits of antenatal education for the childbirth process in Spain. Nurs Res 2010;59:194-202

13. Alves Monteiro MA, Lima Tavares TJ. A prática do grupo de gestantes na efetivação da humanização do parto. Rev RENE 2004;5:73-8.

14. Soto C, Teuber H, Cabrera C, Marín M, Cabrera J, De Costa M, Araneda H. Educación prenatal y su relación con el tipo de parto: una vía hacia el parto natural. Rev Chil Obstet Ginecol 2006;71:98-103. 
15. Thorp JA, Breedlove G. Epidural analgesia in labor: an evaluation of risks and benefits. Birth 1996;23(2):63-83

16. Leighton BL, Halpern SH. The effects of epidural analgesia on labor, maternal, and neonatal outcomes: a systematic review Am J Obstet Gynecol 2002;186(5
Suppl Nature):S69-77.

17. Moran AC, Wahed T, Afsana K. Oxytocin to augment labour during home births: an exploratory study in the urban slums of Dhaka, Bangladesh. BJOG 2010;117:1608-15. 